\title{
Afamin, a novel biomarker: One for all concerns?
}

\author{
Venu Gopal Jonnalagadda ${ }^{1 *}$, Kanchan Choudhary ${ }^{2}$ and Vijay Kranti Matety ${ }^{3}$ \\ ${ }^{1}$ Department of Pharmacology \& Toxicology, National Institute of Pharmaceutical Education and Research, C/O NETES Institute of Technology \& Science, NH- \\ 37, Shantipur, Parli Part, Mirza, Assam - 781125, India \\ ${ }^{2}$ Department of Pharmacology \& Toxicology, Bhupal Nobles' Institute of Pharmaceutical Sciences, Udaipur, Rajasthan- 313001, India
}

During the recent times, metabolic disorders are a burgeoning pandemic in nature stemming to Type 2 diabetes mellitus (T2DM), cardiovascular disease (CVD) including dyslipidemia, hyperglycemia and insulin resistance, abdominal obesity, hypertension and clotting disorders [1]. The prevalence of metabolic syndrome is estimated at more than $30 \%$ in the United States; however, by using the Adult Treatment Panel criteria, prevalence is estimated at about $22 \%$ and increasing with age and depending on sex and ethnic origin $[1,2]$.

Afamin, a human vitamin E binding glycoprotein which was first described in 1994 by Lichtenstein et al. as the fourth member of the human albumin gene family including albumin, a-fetoprotein, and vitamin D-binding protein, is mainly expressed in the liver and secreted into the blood stream, with molecular mass of 87000 Daltons and found on chromosome 4q11-q13 in humans $[1,3]$.

In a three large population-based studies, i.e. from Austria, Northern Italy and Southern Germany were conducted focusing on cardiovascular and metabolic syndrome related effects phenotypes. In this study, the mean afamin concentrations were $63 \pm 15,71 \pm 17$ and $66 \pm 14 \mathrm{mg} / \mathrm{l}$ in the Bruneck, KORA F4 and SAPHIR studies, respectively [4]. The number of metabolic syndrome components increased by $19 \%$ (incidence rate ratio [IRR]: 1.19; 95\% CI:1.16-1.21; $\mathrm{p}$ $<0.0001$ ) with increase of per $10 \mathrm{mg} / \mathrm{l}$ increment in afamin measured at baseline. Also, it was shown that an elevated waist circumference $(\mathrm{OR}=1.72(95 \% \mathrm{CI} 1.64-1.80), \mathrm{p}=5.79 \times 10-123)$ at baseline and $\mathrm{OR}=1.46$ (95\%CI 1.31-1.63), $\mathrm{p}=2.84 \times 10-11$ for change during follow-up) and for elevated fasting glucose concentrations ( $\mathrm{OR}=1.46$ (95\%CI 1.40 1.52), $\mathrm{p}=1.87 \times 10-69$, and $\mathrm{OR}=1.4795 \% \mathrm{CI} 1.35-1.60, \mathrm{p}=1.01 \times 10-18$, respectively [5].

In another study, it was found that afamin concentrations were increased in patients with polycystic ovary syndrome (PCOS), then in controls (odds ratio (OR) for a $10 \mathrm{mg} / \mathrm{ml}$ increase in afamin $=1.3,95 \%$ $\mathrm{CI}=1.08-1.58$ ) [6]. Seeber, et al. reported that afamin concentrations were associated with the presence of metabolic syndrome in young women and serves as prognostic factor for the future development of metabolic syndrome in young women, especially those with insulin resistance and values were as PCOS with IR and PCOS without IR (73.06+/-27.36 mg/L and 64.25+/-17.41 mg/L, p =0.033) [7].

In pregnant women the afamin concentrations were increased in two folds linearly without complications with median afamin serum concentrations of $61.9 \mathrm{mg} / \mathrm{l}, 79.6 \mathrm{mg} / \mathrm{l}$, and $98.6 \mathrm{mg} / \mathrm{l}$ in the first, second, and third trimester. These further warrants investigation of the role of afamin in pregnancy related disorders [8]. Dieplinger $\mathrm{H}$, et al. reported that, concentrations decreased from a median of 70.7 $\mathrm{mg} / \mathrm{L}$ (range, 34.6-116.1 mg/L) in healthy controls to $65.2 \mathrm{mg} / \mathrm{L}$ (range, 20.2-206.6 $\mathrm{mg} / \mathrm{L}$ ) in patients with benign gynecologic diseases to 56.0 $\mathrm{mg} / \mathrm{L}$ (range, 4.7-96.0 mg/L) in ovarian cancer patients $(\mathrm{P}<0.001)$. Considering this, afamin may be an independent diagnostic marker for ovarian cancer as an adjunct marker to cancer antigen 125 (CA 125) [9].

A recently published pooled analysis has shown plasma levels of afamin strongly associated with prediabetes, diabetes mellitus related phenotype characteristics such as IR and both prevalent and incident cases of type 2 diabetes mellitus (OR 1.19 and 1.30, respectively) with afamin concentrations ranged from 61 to $73 \mathrm{mg} / \mathrm{L}$ [10].

The cohort study conducted by Kollerits B, et al. was first analysis conducted in $>20,000$ individuals as a population-based study to establish a phenotypic relationship between afamin concentration and type 2 diabetes mellitus. The main three findings were as mentioned 1) increased concentrations of afamin significantly associated with prediabetes and type 2 diabetes mellitus at baseline and other phenotypes such as insulin resistance (IR) described by HOMAIR and whole body ISI (composite); 2) afamin concentrations were significantly predicted the development of type 2 diabetes independent from major metabolic risk factors or other parameters; 3) afamin showed a significant improvement in model fit and gain in accuracy for incident of type 2 diabetes mellitus. Afamin is primarily expressed in the liver, hence the liver plays an important role in the development of type 2 diabetes mellitus. Thus, the proposed vitamin E binding protein might be having functional relevance to the metabolic syndrome and type 2 diabetes mellitus. According to world health organization (WHO), global diabetes burden doubled since 1980s, finding crucial markers contributing to the development of type 2 diabetes mellitus and is indispensable for rapid identification of affected patients and patients at high risk [10].

In the diagnosis of metabolic disorders, clinicians are increasingly recognizing the need for prognostic biomarkers to predict future occurrence. An afamin concentration has the significant potential to predict future metabolic disorders, but it needs to be confirmed by performing large epidemiological studies including genetic association studies, animal studies and liver cell culture studies, etc. by unraveling structural and functional properties for ligand to control regulation afamin concentration.

*Correspondence to: Venu Gopal Jonnalagadda, Department of Pharmacology \& Toxicology, National Institute of Pharmaceutical Education and Research, C/O NETES Institute of Technology \& Science, NH-37, Shantipur, Parli Part, Mirza, Assam - 781125, India, E-mail: gopalvenu63@gmail.com

Key words: Lysosomal storage diseases; Gaucher disease; $\beta$-Glucocerebrosidase; osseous disease

Received: June 01, 2018; Accepted: June 20, 2018; Published: June 30, 2018 


\section{Conflict of interest}

None

\section{Acknowledgements}

\section{None}

\section{Financial and competing interest}

The author has no relevant affiliations or financial involvement with any organization or entity.

\section{Funding}

This paper was not funded.

\section{References}

1. Kronenberg F, Dieplinger H (2015) Afamin is a promising novel marker for metabolic syndrome and related diseases. Clin Lipidol 10: 207-210.

2. Moore JX, Chaudhary N, Akinyemiju T (2017) Metabolic Syndrome Prevalence by Race/Ethnicity and Sex in the United States, National Health and Nutrition Examination Survey, 1988-2012. Prev Chronic Dis 14: E24. [Crossref]

3. Dieplinger H, Dieplinger B (2015) Afamin-A pleiotropic glycoprotein involved in various disease states. Clin Chim Acta 446: 105-110. [Crossref]
4. Kronenberg F, Kollerits B, Kiechl S, Lamina C, Kedenko L, et al. (2014) Plasma concentrations of afamin are associated with the prevalence and development of metabolic syndrome. Circ Cardiovasc Genet 7: 822-829. [Crossref]

5. Dieplingera H, Kolleritsa B, Laminaa C, Kiechlb S, Willeitb J, Meisingerc C, et al (2014) Plasma concentrations of afamin are associated with the prevalence and development of metabolic syndrome. Atherosclerosis 235: e57.

6. Koninger A, Edimiris P, Koch L, Enekwe A, Lamina C, et al. (2014) Serum concentrations of afamin are elevated in patients with polycystic ovary syndrome. Endocr Connect 3: 120-126. [Crossref]

7. Seeber B, Morandell E, Lunger F, Wildt L, Dieplinger H (2014) Afamin serum concentrations are associated with insulin resistance and metabolic syndrome in polycystic ovary syndrome. Reprod Biol Endocrinol 12: 88. [Crossref]

8. Hubalek M, Buchner H, Mortl MG, Schlembach D, Huppertz B, et al. (2014) The vitamin E-binding protein afamin increases in maternal serum during pregnancy. Clin Chim Acta 434: 41-47. [Crossref]

9. Dieplinger H, Ankerst DP, Burges A, Lenhard M, Lingenhel A, et al. (2009) Afamin and apolipoprotein A-IV: novel protein markers for ovarian cancer. Cancer Epidemiol Biomarkers Prev 18: 1127-1133. [Crossref]

10. Kollerits B, Lamina C, Huth C, Marques-Vidal P, Kiechl S, et al. (2017) Plasma concentrations of Afamin are associated with prevalent and incident Type 2 Diabetes A pooled analysis in more than 20,000 individuals. Diabetes Care 40: 13861393. [Crossref]

Copyright: (C2018 Jonnalagadda VG. This is an open-access article distributed under the terms of the Creative Commons Attribution License, which permits unrestricted use, distribution, and reproduction in any medium, provided the original author and source are credited. 